TRANSACTIONS OF THE

AMERICAN MATHEMATICAL SOCIETY

Volume 350, Number 3, March 1998, Pages 1277-1284

S 0002-9947(98)02102-3

\title{
A CLASSIFICATION THEOREM FOR ALBERT ALGEBRAS
}

\author{
R. PARIMALA, R. SRIDHARAN, AND MANEESH L. THAKUR
}

\begin{abstract}
Let $k$ be a field whose characteristic is different from 2 and 3 and let $L / k$ be a quadratic extension. In this paper we prove that for a fixed, degree 3 central simple algebra $B$ over $L$ with an involution $\sigma$ of the second kind over $k$, the Jordan algebra $J(B, \sigma, u, \mu)$, obtained through Tits' second construction is determined up to isomorphism by the class of $(u, \mu)$ in $H^{1}(k, S U(B, \sigma))$, thus settling a question raised by Petersson and Racine. As a consequence, we derive a "Skolem Noether" type theorem for Albert algebras. We also show that the cohomological invariants determine the isomorphism class of $J(B, \sigma, u, \mu)$, if $(B, \sigma)$ is fixed.
\end{abstract}

\section{INTRODUCTION}

Let $k$ be a field with characteristic different from 2 and 3. Exceptional simple Jordan algebras over $k$ are called Albert algebras. There are rational constructions due to Tits of all Albert algebras over $k$, referred to as the first and the second constructions. We begin by recalling the second construction briefly. Let $L / k$ be a quadratic extension, bar denoting its nontrivial $k$-automorphism. Let $B$ be a degree 3 central simple (associative) algebra over $L$ with an involution $\sigma$ of the second kind over $k$. Let $u$ be a unit of $B$ with $\sigma(u)=u$ and $N(u)=\mu \bar{\mu}, \mu \in L$, where $N$ denotes the reduced norm on $B$. Let $\mathcal{H}(B, \sigma)=\{x \in B \mid \sigma(x)=x\}$. Let $J(B, \sigma, u, \mu)=\mathcal{H}(B, \sigma) \oplus B$ be the Jordan algebra with the multiplication given by

$$
\left.\left(b_{0}, b\right)\left(b_{0}^{\prime}, b^{\prime}\right)=\left(b_{0} \cdot b_{0}^{\prime}+\widetilde{b u \sigma\left(b^{\prime}\right.}\right)+\widetilde{b^{\prime} u \sigma(b)}, \widetilde{b_{0}} b^{\prime}+\widetilde{b_{0}^{\prime}} b+\bar{\mu}\left(\sigma(b) \times \sigma\left(b^{\prime}\right)\right) u^{-1}\right),
$$

where $x . y=\frac{1}{2}(x y+y x), \widetilde{x}=\frac{1}{2}(t(x)-x)$ and

$$
x \times y=x \cdot y-\frac{1}{2} t(x) y-\frac{1}{2} t(y) x+\frac{1}{2}(t(x) t(y)-t(x \cdot y)),
$$

$t$ denoting the reduced trace of $B$. The cubic norm of an element $\left(a_{0}, a\right)$ of $J(B, \sigma, u, \mu)$ is given by

$$
n\left(a_{0}, a\right)=N\left(a_{0}\right)+\mu N(a)+\bar{\mu} N(\sigma(a))-t\left(a_{0} a u \sigma(a)\right) .
$$

For any unit $w \in B$, we have an isomorphism

$$
J(B, \sigma, u, \mu) \longrightarrow J(B, \sigma, w u \sigma(w), N(w) \mu)
$$

given by $\left(a_{0}, a\right) \mapsto\left(a_{0}, a w\right)$. The following converse problem is posed in [P-R].

Question. If $J(B, \sigma, u, \mu) \simeq J\left(B, \sigma, u^{\prime}, \mu^{\prime}\right)$, then does there exist a unit $w \in B$ such that $u^{\prime}=w u \sigma(w), \mu^{\prime}=N(w) \mu$ ?

Received by the editors June 12, 1996.

1991 Mathematics Subject Classification. Primary 17C40.

Key words and phrases. Albert algebras, Tits' constructions.

(C)1998 American Mathematical Society 
In this paper we answer this question in the affirmative ( $\S 2$, Theorem 2.7). As a consequence, we prove a "Skolem Noether" type theorem on extensions of isomorphisms on certain types of simple Jordan subalgebras of an Albert algebra ( 33 , Theorem 3.1). There are cohomological invariants $g_{3} \in H^{3}(k, \mathbb{Z} / 3)$ and $f_{3}, f_{5}$ in $H^{3}(k, \mathbb{Z} / 2)$ and $H^{5}(k, \mathbb{Z} / 2)$ respectively, attached to an Albert algebra $([\mathrm{S}])$. Serre raised the question whether these invariants determine the isomorphism class of the Albert algebra. We indeed show that if $J(B, \sigma, u, \mu)$ and $J\left(B, \sigma, u^{\prime}, \mu^{\prime}\right)$ have the same invariants, then they are isomorphic $(\S 2$, Theorem 2.8$)$.

\section{Coordinatization of a Certain Tits second construction}

Let $L / k$ be a quadratic extension. Let $*$ denote the involution on $M_{3}(L)$ given by $X^{*}=\Gamma^{-1} \bar{X}^{t} \Gamma$, where $\Gamma=\left\langle\gamma_{1}, \gamma_{2}, \gamma_{3}\right\rangle, \gamma_{i} \in k$ with $\gamma_{1} \gamma_{2} \gamma_{3}=1$ and bar denoting the entrywise action of the automorphism bar of $L$. Let $V \in G L_{3}(L)$ with $V^{*}=V$. Suppose further that $\operatorname{det} V=\mu \bar{\mu}$ for some $\mu \in L^{*}$. Then one has the second Tits' construction $J\left(M_{3}(L), *, V, \mu\right)$ with the underlying space $\mathcal{H}\left(M_{3}(L), *\right) \oplus M_{3}(L)$.

The matrix $U=V \Gamma^{-1}$ is hermitian, i.e, $\bar{U}^{t}=U$. Further, $\operatorname{det} U=\operatorname{det} V=$ $\mu \bar{\mu}$. Let $h$ denote the hermitian form on $L^{3}$ given by $h(x, y)=x \bar{U} \bar{y}^{t}$. Then the discriminant of $h$ denoted by disc $h$ is trivial. Let $\psi:\left(\bigwedge^{3} L^{3}, \bigwedge^{3} h\right) \simeq(L,\langle 1\rangle)$ be the trivialization of disc $h$ given by $e_{1} \wedge e_{2} \wedge e_{3} \mapsto \bar{\mu}, e_{i}$ being the standard basis vectors of $L^{3}$. We then have the Cayley algebra (cf. [T]), $C=C\left(L^{3}, h, \psi\right)=L \oplus L^{3}$, with multiplication defined by

$$
(a, v)\left(a^{\prime}, v^{\prime}\right)=\left(a a^{\prime}-h\left(v, v^{\prime}\right), a v^{\prime}+\overline{a^{\prime}} v+\theta\left(v, v^{\prime}\right)\right)
$$

where $\theta$ is defined by the identity

$$
h\left(v^{\prime \prime}, \theta\left(v, v^{\prime}\right)\right)=\psi\left(v^{\prime \prime} \wedge v \wedge v^{\prime}\right),
$$

for all $v, v^{\prime}, v^{\prime \prime} \in L^{3}$. Also, the norm $n_{C}$ is given by $n_{C}(a, v)=n_{L / k}(a)+h(v)$, where $h(v)=h(v, v)$. Then one has the reduced Albert algebra $\mathcal{H}_{3}(C, \Gamma)$ which consists of all $3 \times 3$ matrices

$$
X=\left(\begin{array}{ccc}
\alpha_{1} & c & \gamma_{1}^{-1} \gamma_{3} \bar{b} \\
\gamma_{2}^{-1} \gamma_{1} \bar{c} & \alpha_{2} & a \\
b & \gamma_{3}^{-1} \gamma_{2} \bar{a} & \alpha_{3}
\end{array}\right)
$$

where $\alpha_{i} \in k, a, b, c \in C$, with the multiplication $(X, Y) \mapsto \frac{1}{2}(X Y+Y X)$. Here the bar denotes the involution on $C=L \oplus L^{3}$ given by $\overline{(\alpha, v)}=(\bar{\alpha},-v)$. Note that $\mathcal{H}_{3}(C, \Gamma)$ contains $\mathcal{H}_{3}(L, \Gamma)=\mathcal{H}\left(M_{3}(L), *\right)$ as a Jordan subalgebra. The cubic norm of any element $X$ in $\mathcal{H}_{3}(C, \Gamma)$ as above, is given by

$$
n(X)=\alpha_{1} \alpha_{2} \alpha_{3}-\gamma_{3}^{-1} \gamma_{2} \alpha_{1} n_{C}(a)-\gamma_{1}^{-1} \gamma_{3} \alpha_{2} n_{C}(b)-\gamma_{2}^{-1} \gamma_{1} \alpha_{3} n_{C}(c)+t_{C}((c a) b),
$$

where, for $(\alpha, v) \in C, t_{C}(\alpha, v)=t_{L / k}(\alpha)$. With this notation we have the following.

Theorem 1.1. The map $\Phi: J\left(M_{3}(L), *, V, \mu\right) \rightarrow \mathcal{H}_{3}(C, \Gamma)$, induced by the natural inclusion $\mathcal{H}\left(M_{3}(L), *\right) \hookrightarrow \mathcal{H}_{3}(C, \Gamma)$ and the map $M_{3}(L) \rightarrow \mathcal{H}_{3}(C, \Gamma)$ given by

$$
\left(\begin{array}{l}
\mathbf{a}_{1} \\
\mathbf{a}_{2} \\
\mathbf{a}_{3}
\end{array}\right) \mapsto\left(\begin{array}{ccc}
0 & -\gamma_{1}^{-1} \overline{\mathbf{a}_{3}} & \gamma_{1}^{-1} \overline{\mathbf{a}_{2}} \\
\gamma_{2}^{-1} \overline{\mathbf{a}_{3}} & 0 & -\gamma_{2}^{-1} \overline{\mathbf{a}_{1}} \\
-\gamma_{3}^{-1} \overline{\mathbf{a}_{2}} & \gamma_{3}^{-1} \overline{\mathbf{a}_{1}} & 0
\end{array}\right)
$$

is an isomorphism of Jordan algebras, $\mathbf{a}_{1}, \mathbf{a}_{2}, \mathbf{a}_{3}$ denoting the rows of a matrix in $M_{3}(L)$. 
Proof. Clearly $\Phi$ is an isomorphism of vector spaces. Hence by ([MC-1], p. 507), to check that $\Phi$ is an isomorphism of Jordan algebras, it suffices to check that it preserves the cubic norms and maps identity to identity. It is clear from the definition that $\Phi(1)=1$. Let

$$
\left(A_{0}, A\right)=\left(\left(\begin{array}{ccc}
\alpha_{1} & c & \gamma_{1}^{-1} \gamma_{3} \bar{b} \\
\gamma_{2}^{-1} \gamma_{1} \bar{c} & \alpha_{2} & a \\
b & \gamma_{3}^{-1} \gamma_{2} \bar{a} & \alpha_{3}
\end{array}\right) \quad, \quad\left(\begin{array}{l}
\mathbf{a}_{1} \\
\mathbf{a}_{2} \\
\mathbf{a}_{3}
\end{array}\right)\right)
$$

be any element of $J\left(M_{3}(L), *, V, \mu\right)=\mathcal{H}_{3}(L, \Gamma) \oplus M_{3}(L)$. Equating the norms of $\left(A_{0}, A\right)$ and $\Phi\left(A_{0}, A\right)$ (cf. $\left.\left(*_{1}\right),\left(*_{2}\right)\right)$, we need to verify that

$$
\begin{gathered}
\operatorname{det} A_{0}+\mu \operatorname{det} A+\bar{\mu} \operatorname{det} A^{*}-t\left(A_{0} A V A^{*}\right)=\alpha_{1} \alpha_{2} \alpha_{3} \\
-\gamma_{3}^{-1} \gamma_{2} \alpha_{1} n_{C}\left(a,-\gamma_{2}^{-1} \overline{\mathbf{a}_{1}}\right)-\gamma_{1}^{-1} \gamma_{3} \alpha_{2} n_{C}\left(b,-\gamma_{3}^{-1} \overline{\mathbf{a}_{2}}\right)-\gamma_{2}^{-1} \gamma_{1} \alpha_{3} n_{C}\left(c,-\gamma_{1}^{-1} \overline{\mathbf{a}_{3}}\right) \\
+t_{C}\left(\left(\left(c,-\gamma_{1}^{-1} \overline{\mathbf{a}_{3}}\right)\left(a,-\gamma_{2}^{-1} \overline{\mathbf{a}_{1}}\right)\right)\left(b,-\gamma_{3}^{-1} \overline{\mathbf{a}_{2}}\right)\right)
\end{gathered}
$$

i.e.,

$$
\begin{gathered}
\operatorname{det} A_{0}+\mu \operatorname{det} A+\bar{\mu} \operatorname{det} A^{*}-t\left(A_{0} A V A^{*}\right)=\alpha_{1} \alpha_{2} \alpha_{3}-\gamma_{3}^{-1} \gamma_{2} \alpha_{1} n(a)-\gamma_{1} \alpha_{1} h\left(\overline{\mathbf{a}_{1}}\right) \\
-\gamma_{1}^{-1} \gamma_{3} \alpha_{2} n(b)-\gamma_{2} \alpha_{2} h\left(\overline{\mathbf{a}_{2}}\right)-\gamma_{2}^{-1} \gamma_{1} \alpha_{3} n(c)-\gamma_{3} \alpha_{3} h\left(\overline{\mathbf{a}_{3}}\right)+t_{C}\left(\left(c a-\gamma_{3} h\left(\overline{\mathbf{a}_{3}}, \overline{\mathbf{a}_{1}}\right),\right.\right. \\
\left.\left.-\gamma_{2}^{-1} c \overline{\mathbf{a}_{1}}-\gamma_{1}^{-1} \bar{a} \overline{\mathbf{a}_{3}}+\gamma_{3} \theta\left(\overline{\mathbf{a}_{3}}, \overline{\mathbf{a}_{1}}\right)\right)\left(b,-\gamma_{3}^{-1} \overline{\mathbf{a}_{2}}\right)\right) .
\end{gathered}
$$

We have

$$
\operatorname{det} A_{0}=\alpha_{1} \alpha_{2} \alpha_{3}-\gamma_{3}^{-1} \gamma_{2} n(a)-\gamma_{1}^{-1} \gamma_{3} \alpha_{2} n(b)-\gamma_{2}^{-1} \gamma_{1} \alpha_{3} n(c)+t_{L / k}(c a b) \text {. }
$$

Further, $t_{C}(\alpha, v)=t_{L / k}(\alpha)$, so that we are reduced to verifying the following equality:

$$
\begin{gathered}
\mu \operatorname{det} A+\bar{\mu} \operatorname{det} A^{*}-t\left(A_{0} A V A^{*}\right)=-\alpha_{1} \gamma_{1} h\left(\overline{\mathbf{a}_{1}}\right)-\alpha_{2} \gamma_{2} h\left(\overline{\mathbf{a}_{2}}\right)-\alpha_{3} \gamma_{3} h\left(\overline{\mathbf{a}_{3}}\right) \\
-\gamma_{3} t_{L / k}\left(h\left(\overline{\mathbf{a}_{3}}, \overline{\mathbf{a}_{1}}\right) b\right)-\gamma_{1} t_{L / k}\left(h\left(c \overline{\mathbf{a}_{1}}, \overline{\mathbf{a}_{2}}\right)\right) \\
-\gamma_{2} t_{L / k}\left(h\left(\bar{a} \overline{\mathbf{a}_{3}}, \overline{\mathbf{a}_{2}}\right)\right)+t_{L / k}\left(h\left(\theta\left(\overline{\mathbf{a}_{3}}, \overline{\mathbf{a}_{1}}\right), \overline{\mathbf{a}_{2}}\right)\right) .
\end{gathered}
$$

We first compute $t\left(A_{0} A V A^{*}\right)$. We have

$$
A V A^{*}=A V \Gamma^{-1} \bar{A}^{t} \Gamma=A U \bar{A}^{t} \Gamma=\left(a_{i j}\right) \Gamma,
$$

where $a_{i j}=h\left(\overline{\mathbf{a}_{j}}, \overline{\mathbf{a}_{i}}\right)$. This gives

$$
\begin{aligned}
& t\left(A_{0} A V A^{*}\right)=\alpha_{1} \gamma_{1} h\left(\overline{\mathbf{a}_{1}}\right)+\gamma_{1} c h\left(\overline{\mathbf{a}_{1}}, \overline{\mathbf{a}_{2}}\right)+\gamma_{3} \bar{b} h\left(\overline{\mathbf{a}_{1}}, \overline{\mathbf{a}_{3}}\right)+\gamma_{1} \bar{c} h\left(\overline{\mathbf{a}_{2}}, \overline{\mathbf{a}_{1}}\right) \\
& +\alpha_{2} \gamma_{2} h\left(\overline{\mathbf{a}_{2}}\right)+\gamma_{2} a h\left(\overline{\mathbf{a}_{2}}, \overline{\mathbf{a}_{3}}\right)+\gamma_{3} b h\left(\overline{\mathbf{a}_{3}}, \overline{\mathbf{a}_{1}}\right)+\gamma_{2} \bar{a} h\left(\overline{\mathbf{a}_{3}}, \overline{\mathbf{a}_{2}}\right)+\alpha_{3} \gamma_{3} h\left(\overline{\mathbf{a}_{3}}\right) .
\end{aligned}
$$

Comparing the above with $\left(*_{3}\right)$, it only remains to verify that

$$
\mu \operatorname{det} A+\bar{\mu} \operatorname{det} A^{*}=t_{L / k}\left(h\left(\theta\left(\overline{\mathbf{a}_{3}}, \overline{\mathbf{a}_{1}}\right), \overline{\mathbf{a}_{2}}\right)\right) .
$$

By definition of $\theta$, we have

$$
h\left(\theta\left(\overline{\mathbf{a}_{3}}, \overline{\mathbf{a}_{1}}\right), \overline{\mathbf{a}_{2}}\right)=\overline{\psi\left(\overline{\mathbf{a}_{2}} \wedge \overline{\mathbf{a}_{3}} \wedge \overline{\mathbf{a}_{1}}\right)}=\overline{\bar{\mu} \operatorname{det} \bar{A}}=\mu \operatorname{det} A .
$$

Hence

$$
t_{L / k}\left(h\left(\theta\left(\overline{\mathbf{a}_{3}}, \overline{\mathbf{a}_{1}}\right), \overline{\mathbf{a}_{2}}\right)\right)=\mu \operatorname{det} A+\bar{\mu} \operatorname{det} \bar{A}=\mu \operatorname{det} A+\bar{\mu} \operatorname{det} A^{*} .
$$

For a Jordan algebra $J \simeq \mathcal{H}_{3}(C, \Gamma), C$ is called the coordinate algebra of $J$. Its isomorphism class is uniquely determined by $J$ (cf. [A-J]). 
Corollary 1.2. Let $C$ be any coordinatization of $J\left(M_{3}(L), *, V, \mu\right)$. The norm form $n_{C}$ of $C$ is given by $n_{C}=\operatorname{tr}_{L / k}(\langle 1\rangle \perp h)$, where $h$ is the hermitian form given by the matrix $\overline{V \Gamma^{-1}}$.

Proof. By the uniqueness of the coordinate algebra of a reduced Albert algebra (cf. [A-J]) and by Theorem 1.1 we get $C \simeq C\left(L^{3}, h, \psi\right)$. The corollary now follows (cf. $[\mathrm{T}]$, p. 5124).

\section{Classifichtion of Albert algebras}

Let $k$ be a field with characteristic different from 2 and 3. Let $J$ be an Albert algebra over $k$. Then there exist a 3-fold Pfister form $\phi_{3}$ and a 5 -fold Pfister form $\phi_{5}$ over $k$ such that

$$
Q_{J} \perp \phi_{3} \simeq\langle 2,2,2\rangle \perp \phi_{5},
$$

$Q_{J}$ denoting the trace quadratic form of $J$. This property characterizes $\phi_{3}$ and $\phi_{5}$ up to isomorphism (cf. [S]). Let $G$ be the group of automorphisms of the split Albert algebra over $k$. We have the mod 2 cohomological invariants

$$
f_{3}: H^{1}(k, G) \rightarrow H^{3}(k, \mathbb{Z} / 2)
$$

and

$$
f_{5}: H^{1}(k, G) \rightarrow H^{5}(k, \mathbb{Z} / 2),
$$

defined as the Arason invariants of $\phi_{3}$ and $\phi_{5}$ respectively. If $J \simeq \mathcal{H}_{3}(C, \Gamma)$, then $f_{3}(J)$ is the Arason invariant of $n_{C}$ and $f_{5}(J)$ is the Arason invariant of $n_{C} \otimes$ $\left\langle 1, \gamma_{1}^{-1} \gamma_{2}\right\rangle \otimes\left\langle 1, \gamma_{2}^{-1} \gamma_{3}\right\rangle$. Following a suggestion of Serre, Rost ([R]) constructed a mod 3 invariant

$$
g_{3}: H^{1}(k, G) \rightarrow H^{3}(k, \mathbb{Z} / 3) .
$$

If $J=J(B, \sigma, u, \mu)$ is a second Tits' construction Albert algebra corresponding to a quadratic extension $L$ of $k$, then $g_{3}(J) \in H^{3}(k, \mathbb{Z} / 3)$ maps to $[B] \cup[\mu] \in H^{3}(L, \mathbb{Z} / 3)$; more precisely,

$$
g_{3}(J)=-\operatorname{cor}_{L / k}([B] \cup[\mu]) .
$$

We begin by reviewing a result for Albert algebras belonging to Tits' first construction and which motivated the question posed in the introduction.

Let $k$ be a field of characteristic different from 2 and 3 . Let $A$ be a degree 3 central simple (associative) algebra over $k$ and let $\mu \in k^{*}$. Let $A_{i}=A$ for $i=0,1,2$. On the $k$-vector space $J(A, \mu)=A_{0} \oplus A_{1} \oplus A_{2}$, we define a multiplication by

$$
\begin{gathered}
\left(a_{0}, a_{1}, a_{2}\right)\left(a_{0}^{\prime}, a_{1}^{\prime}, a_{2}^{\prime}\right)=\left(a_{0} \cdot a_{0}^{\prime}+\widetilde{a_{1} a_{2}^{\prime}}+\widetilde{a_{1}^{\prime} a_{2}}, \widetilde{a_{0}} a_{1}^{\prime}+\widetilde{a_{0}^{\prime}} a_{1}\right. \\
\left.+\mu^{-1} a_{2} \times a_{2}^{\prime}, \quad a_{2} \widetilde{a_{0}^{\prime}}+a_{2}^{\prime} \widetilde{a_{0}}+\mu a_{1} \times a_{1}^{\prime}\right),
\end{gathered}
$$

where $x . y, \widetilde{x}, x \times y$ are defined as in the introduction. With this multiplication, $J(A, \mu)$ is an Albert algebra. Given $\mu, \mu^{\prime} \in k^{*}$ with $\mu^{\prime}=N(w) \mu$ for some unit $w \in A$, there is an isomorphism of $J(A, \mu)$ with $J\left(A, \mu^{\prime}\right)$ given by $\left(a_{0}, a_{1}, a_{2}\right) \mapsto$ $\left(w a_{0} w^{-1}, w a_{1}, a_{2} w^{-1}\right)$. Using the Rost invariant for Albert algebras, Petersson and Racine $([\mathrm{P}-\mathrm{R}])$ proved that the converse is true, i.e., if $J(A, \mu) \simeq J\left(A, \mu^{\prime}\right)$, then there exists a unit $w \in A$ such that $\mu^{\prime}=N(w) \mu$.

Let $L / k$ be a quadratic extension and $J\left(B, \sigma, u_{i}, \mu_{i}\right)$ be the Tits' second construction with respect to units $u_{i} \in B, \sigma\left(u_{i}\right)=u_{i}$ and $\mu_{i} \in L^{*}$ with $N\left(u_{i}\right)=\mu_{i} \overline{\mu_{i}}$. The map

$$
(\mathcal{H}(B, \sigma) \oplus B) \otimes_{k} L \rightarrow B_{0} \oplus B_{1} \oplus B_{2},
$$


given by

$$
\left(a_{0}, a\right) \otimes \lambda \mapsto\left(\lambda a_{0}, \lambda a, \bar{\lambda} u_{i} \sigma(a)\right)
$$

where $B_{1}, B_{2}, B_{3}$ are copies of $B$, gives an isomorphism $J\left(B, \sigma, u_{i}, \mu_{i}\right) \otimes_{k} L \simeq$ $J\left(B, \mu_{i}\right), i=1,2$. Thus, in view of the above discussion for algebras of the first kind, we have

Proposition 2.1 (cf. [P-R], p. 205). If $g_{3}\left(J\left(B, \sigma, u_{1}, \mu_{1}\right)\right)=g_{3}\left(J\left(B, \sigma, u_{2}, \mu_{2}\right)\right)$, then $\mu_{1}^{-1} \mu_{2} \in \operatorname{Nrd}\left(B^{*}\right)$.

Proof. Since $g_{3}\left(J\left(B, \sigma, u_{1}, \mu_{1}\right)\right)=g_{3}\left(J\left(B, \sigma, u_{2}, \mu_{2}\right)\right)$, we have $[B] \cup\left[\mu_{1}\right]=[B] \cup\left[\mu_{2}\right]$, which gives $[B] \cup\left[\mu_{1} \mu_{2}^{-1}\right]=0$. Hence the algebra $J\left(B, \mu_{1} \mu_{2}^{-1}\right)$ is reduced $([\mathrm{R}])$. This implies $\mu_{1} \mu_{2}^{-1} \in \operatorname{Nrd}\left(B^{*}\right)$.

Proposition 2.2. If $f_{3}\left(J\left(B, \sigma, u_{1}, \mu_{1}\right)\right)=f_{3}\left(J\left(B, \sigma, u_{2}, \mu_{2}\right)\right)$, then the rank 1 hermitian forms $\left\langle u_{1}\right\rangle$ and $\left\langle u_{2}\right\rangle$ over $(B, \sigma)$ are equivalent.

Proof. In view of ([BF-L]), it suffices to prove this after an odd degree base change of $k$. Let $M$ be an odd degree extension of $k$ such that $B_{M}=B \otimes_{k} M$ is split over $L_{M}=L \otimes_{k} M$. We may therefore assume that $(B, \sigma)=\left(M_{3}(L)\right.$, *), where * is given by $X^{*}=\Gamma^{-1} \bar{X}^{t} \Gamma, \Gamma=\left\langle\gamma_{1}, \gamma_{2}, \gamma_{3}\right\rangle$, with $\gamma_{i} \in k^{*}$. We may further assume that $\gamma_{1} \gamma_{2} \gamma_{3}=1$. Let $u_{1}$ correspond to the matrix $V_{1}$ and $u_{2}$ to the matrix $V_{2}$ in $M_{3}(L)$. Then $\operatorname{det}\left(V_{i}\right)=\mu_{i} \overline{\mu_{i}}, i=1,2$. By Theorem 1.1,

$$
J\left(M_{3}(L), *, V_{1}, \mu_{1}\right) \simeq \mathcal{H}_{3}\left(C_{1}\left(L^{3}, \overline{V_{1} \Gamma^{-1}}, \psi_{1}\right), \Gamma\right)
$$

and

$$
J\left(M_{3}(L), *, V_{2}, \mu_{2}\right) \simeq \mathcal{H}_{3}\left(C_{2}\left(L^{3}, \overline{V_{2} \Gamma^{-1}}, \psi_{2}\right), \Gamma\right),
$$

where $\psi_{i}$ is the trivialization of $\operatorname{disc}\left(\overline{V_{i} \Gamma^{-1}}\right)$ given by $\overline{\mu_{i}}, i=1,2$. We therefore have

$$
f_{3}\left(\mathcal{H}_{3}\left(C_{1}\left(L^{3}, \overline{V_{1} \Gamma^{-1}}, \psi_{1}\right), \Gamma\right)\right)=f_{3}\left(\mathcal{H}_{3}\left(C_{2}\left(L^{3}, \overline{V_{2} \Gamma^{-1}}, \psi_{2}\right), \Gamma\right)\right),
$$

which implies that $n_{C_{1}} \simeq n_{C_{2}}$. So, by Corollary 1.2 ,

$$
\operatorname{tr}_{L / k}\left(\langle 1\rangle \perp h_{1}\right) \simeq \operatorname{tr}_{L / k}\left(\langle 1\rangle \perp h_{2}\right),
$$

where $h_{i}$ is the hermitian form given by the matrix $\overline{V_{i} \Gamma^{-1}}$. Thus, $\operatorname{tr}_{L / k} \circ h_{1} \simeq$ $t r_{L / k} \circ h_{2}$. By a theorem of Jacobson (cf. Appendix 2 of $[\mathrm{M}-\mathrm{H}]$ ), $h_{1} \simeq h_{2}$. So there exists $W \in G L_{3}(L)$ such that $W \overline{V_{1} \Gamma^{-1}} \bar{W}^{t}=\overline{V_{2} \Gamma^{-1}}$, i.e., $\bar{W} V_{1}\left(\Gamma^{-1} W^{t} \Gamma\right)=V_{2}$. Hence $V_{1}$ and $V_{2}$ are hermitian equivalent in $\left(M_{3}(L), *\right)$, which implies that the hermitian forms $\left\langle u_{1}\right\rangle$ and $\left\langle u_{2}\right\rangle$ over $(B, \sigma)$ are equivalent.

Proposition 2.3. Let $u_{1}, u_{2}$ be units in $(B, \sigma)$ with $\sigma\left(u_{i}\right)=u_{i}, n\left(u_{i}\right)=\mu_{i} \overline{\mu_{i}}, \mu_{i} \in$ $L^{*}$. Let $\left\langle u_{1}\right\rangle$ and $\left\langle u_{2}\right\rangle$ be hermitian equivalent over $(B, \sigma)$ and let $\mu_{1}^{-1} \mu_{2}$ be a reduced norm from $B$. Then there exists $w \in B$ such that

$$
u_{2}=w u_{1} \sigma(w), \mu_{2}=n(w) \mu_{1} .
$$

Proof. We introduce an equivalence $\sim$ among the pairs $(u, \mu)$, where $u \in B$ with $\sigma(u)=u$ and $n(u)=\mu \bar{\mu}, \mu \in L^{*}$, as follows: $(u, \mu) \sim\left(u^{\prime}, \mu^{\prime}\right) \Leftrightarrow$ there exists $w \in B$ with $u^{\prime}=w u \sigma(w)$ and $\mu^{\prime}=n(w) \mu$. We show that $\left(u_{1}, \mu_{1}\right) \sim\left(u_{2}, \mu_{2}\right)$.

Let $\mu_{2}=n\left(w_{1}\right) \mu_{1}$. Then $\left(u_{1}, \mu_{1}\right) \sim\left(u^{\prime}, \mu_{2}\right)$, where $u^{\prime}=w_{1} u_{1} \sigma\left(w_{1}\right)$. Since $\left\langle u_{1}\right\rangle$ and $\left\langle u_{2}\right\rangle$ are hermitian equivalent, we get that $\left\langle u^{\prime}\right\rangle$ is hermitian equivalent to $\left\langle u_{2}\right\rangle$. Let $w^{\prime} \in B$ be such that $u_{2}=w^{\prime} u^{\prime} \sigma\left(w^{\prime}\right)$. Now

$$
n\left(u^{\prime}\right)=n\left(w_{1}\right) \overline{n\left(w_{1}\right)} \mu_{1} \overline{\mu_{1}}=\mu_{2} \overline{\mu_{2}}=n\left(u_{2}\right) .
$$


Thus, $n\left(w^{\prime}\right) \overline{n\left(w^{\prime}\right)}=1$. Let $\lambda=n\left(w^{\prime}\right)$. By the following lemma due to Rost, there exist $w^{\prime \prime} \in B$ such that $\lambda=n\left(w^{\prime \prime}\right)$ and $w^{\prime \prime} u_{2} \sigma\left(w^{\prime \prime}\right)=u_{2}$. Thus

$$
\left(u^{\prime}, \mu_{2}\right) \sim\left(u_{2}, \lambda^{-1} \mu_{2}\right) \sim\left(w^{\prime \prime} u_{2} \sigma\left(w^{\prime \prime}\right), n\left(w^{\prime \prime}\right) \lambda^{-1} \mu_{2}\right)=\left(u_{2}, \mu_{2}\right) .
$$

Therefore, $\left(u_{1}, \mu_{1}\right) \sim\left(u_{2}, \mu_{2}\right)$.

Lemma 2.4 (Rost). Let $L / k$ be a quadratic extension. Let $A$ be a degree 3 central simple (associative) algebra over $L$ with an involution $\sigma$ of the second kind. Let $x \in L^{*}$ be such that $x \sigma(x)=1$. Assume that $x=N(a)$ for some $a \in A$. Then there exists $a^{\prime} \in A$ with $x=N\left(a^{\prime}\right)$ and $a^{\prime} \sigma\left(a^{\prime}\right)=1$.

In fact, we have the following more general lemma due to Suresh, the proof of which uses the following result of Merkurjev on norm principle for unitary groups.

Lemma 2.5 (Merkurjev). Let $x \in L^{*}$. Then $x=N\left(v \sigma(v)^{-1}\right)$ for some $v \in A^{*}$ if and only if there exists $u \in A^{*}$ with $u \sigma(u)=1$ and $x=N(u)$.

Proof. (cf. Theorem 5.1.3 of [BF-P]).

Lemma 2.6 (Suresh). Let $L / k$ be a quadratic extension and $A$ a central simple (associative) algebra over $L$ of odd degree with an involution of the second kind. Let $x \in L$ be such that $x \bar{x}=1$. If $x$ is the reduced norm of some element of $A$, then there exists an element $u \in A^{*}$ such that $u \sigma(u)=1$ and $N(u)=x$.

Proof. Let $x \in L^{*}$ be such that $x \bar{x}=1$ and $x=N(v)$ for some $v \in A^{*}$. Then there exists $y \in L^{*}$ such that $x=y(\bar{y})^{-1}$. Since $\sigma$ is the identity on $k$, for $\lambda \in k^{*}$ we have $x=\lambda y(\overline{\lambda y})^{-1}$. Therefore, by Lemma 2.5 , it is enough to show that $\lambda y$ is a reduced norm of some element of $A^{*}$ for some $\lambda \in k^{*}$. Let $[A: L]=(2 r+1)^{2}$. We have

$$
\begin{aligned}
N\left(v^{r} \bar{y}\right) & =N(v)^{r}(\bar{y})^{2 r+1} \quad\left(\text { since } \sigma(y) \in L^{*}\right) \\
& =x^{r}(\bar{y})^{2 r+1} \\
& =\left(y^{r}(\bar{y})^{-r}\right)(\bar{y})^{2 r+1} \\
& =(y \bar{y})^{r} \bar{y}
\end{aligned}
$$

Let $\lambda=(y \bar{y})^{r} \in k$. Then it follows that $\lambda \bar{y}$ is a reduced norm from $A^{*}$, and hence $\lambda y$ is a reduced norm from $A^{*}$. This completes the proof of lemma.

Theorem 2.7. Let $k$ be a field with characteristic different from 2 and 3. Let $L / k$ be a quadratic extension, $B$ a degree 3 central simple algebra over $L$ with an involution $\sigma$ of the second kind. Let $u_{i}, i=1,2$, be units in $B$ with $\sigma\left(u_{i}\right)=u_{i}$ and $n\left(u_{i}\right)=\mu_{i} \overline{\mu_{i}}, \mu_{i} \in L^{*}$. Then $J\left(B, \sigma, u_{1}, \mu_{1}\right) \simeq J\left(B, \sigma, u_{2}, \mu_{2}\right)$ if and only if there is $w \in B$ with $u_{2}=w u_{1} \sigma(w)$ and $\mu_{2}=n(w) \mu_{1}$.

Proof. If $u_{2}=w u_{1} \sigma(w)$ and $\mu_{2}=n(w) \mu_{1}$ then, as was remarked in the introduction, the map $\left(a_{0}, a\right) \mapsto\left(a_{o}, a w\right)$ gives an isomorphism of $J\left(B, \sigma, u_{1}, \mu_{1}\right)$ with $J\left(B, \sigma, u_{2}, \mu_{2}\right)$.

Conversely, suppose $J_{1}=J\left(B, \sigma, u_{1}, \mu_{1}\right) \simeq J_{2}=J\left(B, \sigma, u_{2}, \mu_{2}\right)$. Then $f_{3}\left(J_{1}\right)=$ $f_{3}\left(J_{2}\right)$ and $g_{3}\left(J_{1}\right)=g_{3}\left(J_{2}\right)$. The theorem now follows from Propositions 2.1, 2.2 and 2.3 .

Theorem 2.8. Let $J=J(B, \sigma, u, \mu), J^{\prime}=J\left(B, \sigma, u^{\prime}, \mu^{\prime}\right)$ be Albert algebras such that $f_{3}(J)=f_{3}\left(J^{\prime}\right)$ and $g_{3}(J)=g_{3}\left(J^{\prime}\right)$. Then $J \simeq J^{\prime}$.

Proof. The proof follows from Propositions 2.1, 2.2, 2.3 and the 'if' part of Theorem 2.7. 
Remark. Theorem 2.7 asserts that the map $H^{1}(k, S U(B, \sigma)) \rightarrow H^{1}\left(k, F_{4}\right)$, on the Galois cohomology, corresponding to the second Tits' construction, is injective.

\section{A Skolem Noether type theorem}

In this section we prove the following "Skolem Noether" type theorem.

Theorem 3.1. Let $L / k$ be a quadratic field extension. Let $(B, \sigma),\left(B^{\prime}, \sigma^{\prime}\right)$ be degree 3 central simple algebras over $L$ with involutions of the second kind over $k$. Let $\mathcal{H}(B, \sigma), \mathcal{H}\left(B^{\prime}, \sigma^{\prime}\right)$ denote the 9-dimensional Jordan algebras over $k$ associated to the symmetric elements in $(B, \sigma),\left(B^{\prime}, \sigma^{\prime}\right)$ respectively. Suppose that $\mathcal{H}(B, \sigma)$ and $\mathcal{H}\left(B^{\prime}, \sigma^{\prime}\right)$ are subalgebras of an Albert algebra $J$ over $k$ and $\alpha: \mathcal{H}(B, \sigma) \simeq \mathcal{H}\left(B^{\prime}, \sigma^{\prime}\right)$ is an isomorphism of Jordan algebras. Then $\alpha$ extends to an automorphism of $J$.

For the proof we need some lemmas (which are also consequences of a more general result proved by Jacobson [J], p. 210, Theorem 11. However, we give direct proofs for the sake of completeness.)

Lemma 3.2. Let $L / k$ be a quadratic field extension. Let $B$ be a degree 3 central simple algebra with an involution $\sigma$ of the second kind. Let $\alpha: \mathcal{H}(B, \sigma) \rightarrow \mathcal{H}(B, \sigma)$ be an automorphism of Jordan algebras. Then $\alpha$ is the restriction of an isomorphism $\widetilde{\alpha}:(B, \sigma) \simeq(B, \sigma)$ or $\widetilde{\alpha}:(B, \sigma) \simeq\left(B^{o p}, \sigma\right)$ of associative algebras with involutions.

Proof. Consider the map $\alpha \otimes 1: \mathcal{H}(B, \sigma) \otimes_{k} L \simeq \mathcal{H}(B, \sigma) \otimes_{k} L$. The map $\psi$ : $\mathcal{H}(B, \sigma) \otimes_{k} L \rightarrow B$, given by $(x, \lambda) \mapsto x \lambda$, is an isomorphism of Jordan algebras over $L$. Let $\widetilde{\alpha}=\psi \circ(\alpha \otimes 1) \circ \psi^{-1}: B \simeq B$. Then $\widetilde{\alpha}$ restricts on $\mathcal{H}(B, \sigma)$ to $\alpha$. By a theorem of Ancochea $([\mathrm{A}]), \widetilde{\alpha}$ is an automorphism or an anti-automorphism of the algebra $B$. We show that $\widetilde{\alpha}$ commutes with $\sigma$. For $x \in \mathcal{H}(B, \sigma), \sigma(x)=x$, so that

$$
\widetilde{\alpha} \sigma(x)=\widetilde{\alpha}(x)=\alpha(x)=\sigma \widetilde{\alpha}(x) .
$$

Let $L=k(j)$ with $j^{2} \in k$. Then, $\widetilde{\alpha} \sigma(j)=\widetilde{\alpha}(-j)=-j=\sigma \widetilde{\alpha}(j)$. Since $\mathcal{H}(B, \sigma)$ generates $B$ as an $L$-algebra, it follows that $\widetilde{\alpha}$ commutes with $\sigma$ on the whole of $B$. Hence $\widetilde{\alpha}$ has the required properties.

Theorem 3.3. Let $B$ be a central simple $L$-algebra of degree 3 with involutions $\sigma, \sigma^{\prime}$ of the second kind. Let $Q_{\sigma}, Q_{\sigma^{\prime}}$ denote the restrictions to $\mathcal{H}(B, \sigma)$ and $\mathcal{H}\left(B, \sigma^{\prime}\right)$, respectively, of the trace form of $B$. Then the involutions $\sigma$ and $\sigma^{\prime}$ are isomorphic if and only if $Q_{\sigma}$ and $Q_{\sigma^{\prime}}$ are isometric.

Proof. See [H-K-R-T], Proposition 4.

Lemma 3.4. Let $L / k$ be a quadratic extension of $k$. Let $(B, \sigma),\left(B^{\prime}, \sigma^{\prime}\right)$ be degree 3 central simple (associative) algebras over $L$ with involutions of the second kind. If $\alpha: \mathcal{H}(B, \sigma) \simeq \mathcal{H}\left(B^{\prime}, \sigma^{\prime}\right)$ is an isomorphism of Jordan algebras, then there exists an isomorphism $\widetilde{\alpha}:(B, \sigma) \rightarrow\left(B^{\prime}, \sigma^{\prime}\right)$ or $\widetilde{\alpha}:(B, \sigma) \rightarrow\left(B^{\prime}\right.$ op,$\left.\sigma^{\prime}\right)$ of associative algebras with involutions, which restricts to $\alpha$.

Proof. The isomorphism $\alpha$ extends to a Jordan algebra isomorphism

$$
\mathcal{H}(B, \sigma) \otimes_{k} L \simeq \mathcal{H}\left(B^{\prime}, \sigma^{\prime}\right) \otimes_{k} L,
$$

which in turn gives an isomorphism of the Jordan algebra $B$ with $B^{\prime}$. By a theorem of Ancochea, $B$ is isomorphic or anti-isomorphic to $B^{\prime}$. Replacing $B^{\prime}$ by $B^{\prime o p}$, if necessary, we may assume that $B$ is isomorphic to $B^{\prime}$. In view of the fact that $\alpha$ is an isomorphism of Jordan algebras, we get that $Q_{\sigma} \simeq Q_{\sigma^{\prime}}$. By Theorem 3.3 and 
the fact that $B$ is isomorphic to $B^{\prime}$ we conclude that there exists an isomorphism $\widetilde{\beta}:(B, \sigma) \simeq\left(B^{\prime}, \sigma^{\prime}\right)$ or $(B, \sigma) \simeq\left(B^{\prime o p}, \sigma^{\prime}\right)$ of associative algebras with involutions. Thus $\widetilde{\beta}$ restricts to $\beta: \mathcal{H}(B, \sigma) \simeq \mathcal{H}\left(B^{\prime}, \sigma^{\prime}\right)$. Consider $\beta^{-1} \circ \alpha: \mathcal{H}(B, \sigma) \rightarrow \mathcal{H}(B, \sigma)$. By Lemma 3.2, there exists $\gamma:(B, \sigma) \simeq(B, \sigma)$ or $(B, \sigma) \simeq\left(B^{o p}, \sigma\right)$, which restricts to $\beta^{-1} \circ \alpha$. Let $\widetilde{\alpha}=\widetilde{\beta} \circ \gamma$. Then $\widetilde{\alpha}$ satisfies the requirements in the lemma.

Proof of Theorem 3.1. By Lemma 3.4 there is an isomorphism $\widetilde{\alpha}:(B, \sigma) \rightarrow\left(B^{\prime}, \sigma^{\prime}\right)$ or $(B, \sigma) \rightarrow\left(B^{\prime o p}, \sigma^{\prime}\right)$ which restricts to $\alpha$. By ([MC-2]), there are isomorphisms

$$
\phi_{1}: J(B, \sigma, u, \mu) \simeq J, \phi_{2}: J\left(B^{\prime}, \sigma^{\prime}, u^{\prime}, \mu^{\prime}\right) \simeq J
$$

for suitable $u, \mu, u^{\prime}, \mu^{\prime}$, which restrict to the inclusions of $\mathcal{H}(B, \sigma)$ and $\mathcal{H}\left(B^{\prime}, \sigma^{\prime}\right)$ in $J$. We have an isomorphism $J(\widetilde{\alpha}): J(B, \sigma, u, \mu) \simeq J\left(B^{\prime}, \sigma^{\prime}, \alpha(u), \mu\right)$ given by $\left(a_{0}, a\right) \mapsto\left(\alpha\left(a_{0}\right), \alpha(a)\right)$. But $J\left(B^{\prime}, \sigma^{\prime}, \alpha(u), \mu\right) \simeq J\left(B^{\prime}, \sigma^{\prime}, u^{\prime}, \mu^{\prime}\right)$, since both are isomorphic to $J$. By Theorem 2.2 of $\S 2$, there exists $w^{\prime} \in B^{\prime}$ such that $u^{\prime}=$ $w^{\prime} \alpha(u) \sigma^{\prime}\left(w^{\prime}\right), \mu^{\prime}=N\left(w^{\prime}\right) \mu$. Let $\psi: J\left(B^{\prime}, \sigma^{\prime}, \alpha(u), \mu\right) \simeq J\left(B^{\prime}, \sigma^{\prime}, u^{\prime}, \mu^{\prime}\right)$ be given by $\left(a_{0}^{\prime}, a^{\prime}\right) \mapsto\left(a_{0}^{\prime}, a^{\prime} w^{\prime}\right)$. Then $\psi$ restricts to the identity map on $\mathcal{H}\left(B^{\prime}, \sigma^{\prime}\right)$. Let $\phi=\phi_{2} \circ \psi \circ J(\widetilde{\alpha}) \circ \phi_{1}^{-1}$. Then for $x \in \mathcal{H}(B, \sigma)$, we have $\phi(x)=\phi_{2} \psi(\alpha(x))=\alpha(x)$. Thus $\phi$ is an automorphism of $J$ extending $\alpha$.

\section{REFERENCES}

[A] G. Ancochea.On semi-automorphisms of division algebras, Ann. Math. 48 (1947), 147-154. MR 8:310c

[A-J] A. A. Albert, N. Jacobson. On reduced exceptional simple Jordan algebras, Ann. Math.(2)66(1957), 400-417. MR 19:527b

[BF-L] E. Bayer-Fluckiger, H. W. Lenstra Jr. Forms in odd degree extensions and self-dual normal bases, Amer. J. Math. 112(1990), 359-373. MR 91h:11030

[BF-P] E. Bayer-Fluckiger, R. Parimala. Galois cohomology of the classical groups over fields of cohomological dimension $\leq 2$, Invent. Math. 122(1995), 195-229. MR 96i:11042

[H-K-R-T] D. Haile, M. A. Knus, M. Rost, J. P. Tignol. Algebras of odd degree with involution, trace forms and Kummer extensions, Israel J. Math. 19 (1996), 299-340. CMP 97:08

[J] N. Jacobson. Structure and representations of Jordan algebras, A. M. S. Colloquium Publications, Volume 39. A. M. S. Providence, Rhode Island, 1968. MR 40:4330

[MC-1] K. McCrimmon. The Freudenthal-Springer-Tits constructions of exceptional Jordan algebras, Trans. Amer. Math. Soc. 139(1969), 495-510. MR 39:276

[MC-2] K. McCrimmon. The Freudenthal-Springer-Tits constructions revisited, Trans. Amer. Math. Soc. 148(1970), 293-314. MR 42:6064

[M-H] J. Milnor, D. Husemoller. Symmetric Bilinear Forms, Ergebnisse der Mathematik und ihrer Grenzgebiete, Band 73. Springer-Verlag, Berlin, Heidelberg, New York, 1973. MR 58:22129

[P-R] H. P. Petersson, M. Racine. Albert Algebras, Proceedings of a conference on Jordan algebras, Oberwolfach, 1992, de Gruyter, Berlin, 1994, pp. 197-207. MR 95k:17043

[R] M. Rost. A (mod 3) invariant for exceptional Jordan algebras, C.R. Acad. Sci. Paris Sér. I Math. 313 (1991), 823-827. MR 92j:19002

[S] J. P. Serre. Cohomologie Galoisienne: progrès et problèmes, Seminaire Bourbaki 1993/94, Exposé 763, Astérisque, no. 227, Soc. Math. France, Paris, 1995, pp. 229-257. MR 97d:11063

[T] M. L. Thakur. Cayley algebra bundles on $A_{k}^{2}$ revisited, Comm. Alg, 23(13) (1995), 5119-5130. MR 97a:17006

School of Mathematics, Tata Institute of Fundamental Research, Homi Bhabha RoAD, BOMBaY-5, INDia

E-mail address: parimala/sridhar/maneesh@tifrvax.tifr.res.in 